\title{
APPLICATIONS OF SPHERICAL DESIGNS TO BANACH SPACE THEORY
}

\author{
HERMANN KÖNIG \\ Mathematisches Seminar, Universität Kiel, 24098 Kiel, Germany \\ E-mail: hkoenig@math.uni-kiel.de
}

\begin{abstract}
Spherical designs constitute sets of points distributed on spheres in a regular way. They can be used to construct finite-dimensional normed spaces which are extreme in some sense: having large projection constants, big or small Banach-Mazur distance to Hilbert spaces or $\ell_{p}$-spaces. These examples provide concrete illustrations of results obtained by more powerful probabilistic techniques which, however, do not exhibit explicit examples. We give a survey of such constructions where the geometric invariants can be estimated quite precisely.
\end{abstract}

1. Introduction. Dvoretzky's theorem and the study of Hilbertian subspaces of highdimensional Banach spaces has been the subject of intensive investigation which typically involve probabilistic techniques and the concentration of measure phenomenon, [D], [MS]. Below a certain dimension, "typical" subspaces are Hilbertian whereas for higher dimensions, the maximal Banach-Mazur distance to Hilbert spaces can now be estimated quite precisely, at least for classical spaces like the $\ell_{p}$-spaces. Very often such spaces are very badly complemented and thus have very large projection constants, e.g. for Kashin's subspaces of $\ell_{1}$, [Ka], or Gluskin's spaces yielding the correct diameter estimate for the Banach-Mazur compactum, [G]. "Typical" subspaces here is meant in a probabilistic way, i.e. with respect to the Haar measure on the Graßmann manifold of $n$-dimensional subspaces of $N$-dimensional spaces where $n<N$.

In this note, we present some more explicit constructions of Hilbertian subspaces of classical Banach spaces and of spaces with large projection constants which rely on the existence of regular point distributions on spheres-spherical designs-instead of random point set distributions.

As for notation, $\ell_{p}^{N}$ denotes $\mathbb{K}^{N}, \mathbb{K} \in\{\mathbb{R}, \mathbb{C}\}$, equipped with the norm

$$
\|x\|_{p}=\left(\sum_{j=1}^{N}\left|x_{j}\right|^{p}\right)^{1 / p}, \quad x=\left(x_{j}\right)_{j=1}^{N} \in \mathbb{K}^{N} .
$$

2000 Mathematics Subject Classification: Primary 46B20.

The paper is in final form and no version of it will be published elsewhere. 
The Banach-Mazur distance between two isomorphic Banach spaces $X$ and $Y$ is defined to be

$$
d(X, Y)=\inf \left\{\|T\|\left\|T^{-1}\right\| \mid T: X \rightarrow Y \text { linear isomorphism }\right\} .
$$

Let $n, k, N \in \mathbb{N}$. A spherical design of order $2 k$ in $n$ variables with $N$ nodes consists of $N$ points on the euclidean unit sphere $x_{1}, \ldots, x_{N} \in S^{n-1}(\mathbb{K})$ and a discrete probability measure on them $, \mu_{1}, \ldots, \mu_{N}>0, \sum_{s=1}^{N} \mu_{s}=1$ such that all even polynomials of degree $2 k$ in $n$ variables $\mathcal{P}_{2 k, n}^{e v e n}$ are integrated exactly over the normalized Haar measure $\sigma$ on the sphere $S^{n-1}$ by the quadrature formula defined by $\left(x_{s}, \mu_{s}\right)$, i.e.

$$
\sum_{s=1}^{N} \mu_{s} p\left(x_{s}\right)=\int_{S^{n-1}} p(y) d \sigma(y), \quad p \in \mathcal{P}_{2 k, n}^{\text {even }} .
$$

For $\mathbb{K}=\mathbb{C}, \mathbb{C}^{n} \simeq \mathbb{R}^{2 n}$ are identified for the purpose of integration; "even polynomials" then means polynomials in $(z, \bar{z})$ which are homogeneous of degree $k$ each in $z$ and in $\bar{z}$. It suffices to have (1.1) for polynomials which are homogeneous of the highest degree $2 k$, since lower order $(2 \ell)$-homogeneous parts can be multiplied by $1=(y, y)^{k-\ell}$ to get the same degree $(2 k)$ of homogenity when restricted to $S^{n-1}$. Spherical designs in the classical sense of Delsarte, Goethals and Seidel [DGS] are more restricted having $\mu_{s}=1 / N$ but (1.1) is more suitable for our purpose. In the case of $k=1$, polynomials of degree 2 , take $p(y)=(y \otimes y)_{i j}=y_{i} y_{j}$ for $y=\left(y_{j}\right)_{j=1}^{n} \in \mathbb{K}^{n}$. Since $\int_{S^{n-1}} p(y) d \sigma(y)=1 / n \delta_{i j}$, a spherical design of degree 2 just means

$$
n \sum_{s=1}^{N} \mu_{s} x_{s} \otimes x_{s}=\operatorname{Id}_{\mathbb{K}^{n}} .
$$

John's theorem typically yields such points which as contact points of a norm additionally satisfy $\left\|x_{s}\right\|_{X}=\left\|x_{s}\right\|_{X^{*}}=1$. The points $\left(x_{s}\right)$ in (1.2) are orthogonal projections of an orthonormal basis in $\ell_{2}^{N}$ onto a suitable $n$-dimensional subspace:

$$
P:=\left(\mu_{s}\left\langle x_{s}, x_{t}\right\rangle\right)_{s, t=1}^{N}: \mathbb{K}^{N} \rightarrow \mathbb{K}^{N}
$$

Here $\mathbb{K}^{n}$ should be thought of being imbedded into $\mathbb{K}^{N}$ by $x \mapsto\left(\mu_{s}\left\langle x, x_{s}\right\rangle\right)_{s=1}^{N}$.

2. Hilbertian subspaces of $\ell_{p}$-spaces. Let $\varepsilon>0$ and $1 \leq p<\infty$. By Dvoretzky's theorem for $\ell_{p}$-spaces, given $n \in \mathbb{N}$ there exists $c_{p}>0$ such that if $N \in \mathbb{N}$ satisfies

$$
N \geq\left\{\begin{array}{cc}
c_{p} n & 1 \leq p \leq 2 \\
c_{p} n^{p / 2} & 2 \leq p<\infty
\end{array}\right\},
$$

the $N$-dimensional space $\ell_{p}^{N}$ contains almost Hilbertian subspaces $Y_{n} \subset \ell_{p}^{N}$ of $\operatorname{dim} Y_{n}=$ $n, d\left(Y_{n}, \ell_{2}^{n}\right) \leq 1+\varepsilon,[\mathrm{FLM}]$. We say that $\ell_{2}^{n}$ embeds $(1+\varepsilon)$-isomorphically into $\ell_{p}^{N}$.

We consider the case of isometric imbeddings, i.e. $\varepsilon=0$ and $n \geq 2$. Do isometric imbeddings of $\ell_{2}^{n}$ into $\ell_{p}^{N}$ exsist? The answer is yes if and only if $p$ is an even integer, $p=2 k \in 2 \mathbb{N}$. That $p$ needs to be even can be found in Lyubich's paper [L], that isometric imbeddings of $\ell_{2}^{n}$ into $\ell_{p}^{N}$ exist for $p=2 k$ was shown by Gromov, Milman [M]. In fact, even in the infinite-dimensional case, isometric imbeddings of $\ell_{2}^{2}$ into $\ell_{p}$ only exist if $p=2 k \in 2 \mathbb{N}$ as was proved by Delbaen, Jarchow and Pełczyński [DJP]. They also prove 
that general $n$-dimensional subspaces of $L_{p}$ are imbedded isometrically into $\ell_{2 k}^{N}$ if $N$ is sufficiently large.

The following proposition states the equivalence of imbedding $\ell_{2}^{n}$ isometrically into $\ell_{2 k}^{N}$ with the existence of appropriate spherical designs. It is a generalization of results of Delsarte, Goethals and Seidel [DGS] and Reznick [R] and was stated in [K]. The equivalence $(1) \Leftrightarrow(2)$ was also obtained by Lyubich and Vaserstein [LV].

Proposition 1. Let $n, k, N \in \mathbb{N}$. Then the following are equivalent:

(1) There exists an isometric imbedding of $\ell_{2}^{n}$ into $\ell_{2 k}^{N}$.

(2) There exists a spherical design $\left(x_{s}, \mu_{s}\right)_{s=1}^{N}$ of order $2 k$ in $n$ variables with $N$ nodes.

(3) There exist points $x_{1}, \ldots, x_{N} \in S^{n-1}(\mathbb{K})$ and $\mu_{s}>0, \sum_{s=1}^{N} \mu_{s}=1$ such that

$$
\sum_{s, t=1}^{N} \mu_{s} \mu_{t}\left|\left\langle x_{s}, x_{t}\right\rangle\right|^{2 k}=\int_{S^{n-1}} \int_{S^{n-1}}|\langle x, y\rangle|^{2 k} d \sigma(x) d \sigma(y)=: c_{n k} .
$$

Condition (2.1) gives a criterion to check whether $\left(x_{s}, \mu_{s}\right)$ is a spherical design which defines an imbedding. We sketch the important directions $(3) \Rightarrow(2)$ and $(2) \Rightarrow(1)$ in the real case; $(1) \Rightarrow(3)$ is not difficult either.

Proof. $(3) \Rightarrow(2)$. For $x \in \mathbb{R}^{n}$ and $\ell \in N$, denote $x^{\otimes \ell}=x \otimes \cdots \otimes x \in \mathbb{R}^{n^{\ell}}$ the $\ell$-fold tensor product. Then $\left\langle x^{\otimes \ell}, y^{\otimes \ell}\right\rangle=\langle x, y\rangle^{\ell}$ which for even $\ell$ coincides with $|\langle x, y\rangle|^{\ell}$. Given points $x_{s} \in S^{n-1}$ and $\mu_{s}>0, \sum_{s=1}^{N} \mu_{s}=1$, form the tensor

$$
\xi:=\sum_{s=1}^{N} \mu_{s} x_{s}^{\otimes 2 k}-\int_{S^{n-1}} y^{\otimes 2 k} d \sigma(y) \in \mathbb{R}^{n^{2 k}} .
$$

Evaluating the scalar product $0 \leq\langle\xi, \xi\rangle$ in $\mathbb{R}^{n^{2 k}}$, we find

$0 \leq \sum_{s, t=1}^{N} \mu_{s} \mu_{t}\left|\left\langle x_{s}, x_{t}\right\rangle\right|^{2 k}-2 \sum_{s=1}^{N} \mu_{s} \int_{S^{n-1}}\left|\left\langle x_{s}, y\right\rangle\right|^{2 k} d \sigma(y)+\int_{S^{n-1}} \int_{S^{n-1}}|\langle x, y\rangle|^{2 k} d \sigma(y)$.

The integral in the second sum does not depend on $s$ since $\left|x_{s}\right|=1$ and $\sigma$ is rotation invariant; it equals $c_{n k}$ as defined in (2.1). Hence

$$
0 \leq \sum_{s, t=1}^{N} \mu_{s} \mu_{t}\left|\left\langle x_{s}, x_{t}\right\rangle\right|^{2 k}-c_{n k} \quad \text { (Sidelnikov's inequality). }
$$

Thus (2.1) means that $\xi=0$. In coordinates this means that all homogeneous polynomials of degree $2 k$ are integrated exactly by the quadrature formula defined by $\left(x_{s}, \mu_{s}\right)$ and $(2)$ follows from this.

$(2) \Rightarrow(1)$. Given the design $\left(x_{s}, \mu_{s}\right)$, consider the imbedding

$$
i: \ell_{2}^{n} \rightarrow \ell_{2 k}^{N}, \quad x \mapsto\left(\left(\mu_{s} / c_{n k}\right)^{1 / 2 k}\left\langle x, x_{s}\right\rangle\right)_{s=1}^{N},
$$

Denoting $p_{x}(y):=\langle x, y\rangle^{2 k}$ for any fixed $x \in \mathbb{R}^{n}$, we have

$$
\|i x\|_{2 k}^{2 k}=\sum_{j=1}^{N}\left(\mu_{s} / c_{n k}\right) p_{x}\left(x_{s}\right)=\left(1 / c_{n k}\right) \int_{S^{n-1}} p_{x}(y) d \sigma(y)=|x|^{2 k}
$$

i.e. $i$ is isometric. 
REMARK. If $\left(x_{s}, \mu_{s}\right)$ are given explicitly, the imbedding $i$ is defined explicitly as the proof shows. The usefulness of (2.1) is best illustrated by a few examples.

\section{EXAMPLES.}

(1) $\mathbb{K}=\mathbb{R}, n=2, N=k+1$. Take $x_{s}=\exp \left(\frac{2 \pi i}{N} s\right)_{s=0}^{k} \in \mathbb{C} \simeq \mathbb{R}^{2}, \mu_{s}=1 / N$. It is easy to check that (2.1) holds, so $\ell_{2}^{2} \hookrightarrow \ell_{2 k}^{k+1}$ imbeds isometrically. In particular $i: \ell_{2}^{2} \hookrightarrow \ell_{4}^{3}$ with $i\left(\ell_{2}^{2}\right)=\left\{y \in \ell_{4}^{3} \mid \sum_{s=1}^{3} y_{s}=0, y=\left(y_{s}\right)_{s=1}^{3}\right\}$.

(2) $\mathbb{K}=\mathbb{C}, n=2, k=2, N=4: \ell_{2}^{2}(\mathbb{C}) \hookrightarrow \ell_{4}^{4}(\mathbb{C})$ by using $\mu_{s}=1 / 4$ and the vectors $x_{1}=(1,0), x_{s}=\frac{1}{\sqrt{3}}\left(1, \sqrt{2} \omega^{s-2}\right), s=2,3,4$ with $\omega=\exp (2 \pi i / 3)$.

(3) $\mathbb{K}=\mathbb{R}, n=24, k=5, N=98280$ : this uses vectors of the Leech lattice, see [R] or [DGS].

It is of course of interest to determine the minimal dimension $N$ of the $\ell_{p}^{N}$-space, $p=2 k$, where $\ell_{2}^{n}$ imbeds isometrically into; for quadrature formulas this means the minimal number of nodes.

Definition. $N(n, k):=\min \left\{N \in \mathbb{N} \mid \ell_{2}^{n} \hookrightarrow \ell_{2 k}^{N}\right.$ imbeds isometrically $\}$.

Proposition 2. Let $2 \leq n, k \in \mathbb{N}$. Then

$$
\begin{aligned}
\left(\begin{array}{c}
n+k-1 \\
k
\end{array}\right) \leq N(n, k) \leq\left(\begin{array}{c}
n+2 k-1 \\
2 k
\end{array}\right), \quad \mathbb{K}=\mathbb{R}, \\
\left(\begin{array}{c}
n+\left[\frac{k+1}{2}\right]-1 \\
{\left[\frac{k+1}{2}\right]}
\end{array}\right)\left(\begin{array}{c}
n+\left[\frac{k}{2}\right]-1 \\
{\left[\frac{k}{2}\right]}
\end{array}\right) \leq N(n, k) \leq\left(\begin{array}{c}
n+k-1 \\
k
\end{array}\right), \quad \mathbb{K}=\mathbb{C} .
\end{aligned}
$$

For the upper bound, see [M], for the lower e.g. in the real case [R]. Note that $\left(\begin{array}{c}n+k-1 \\ k\end{array}\right)$ is the dimension of the (real) polynomials in $n$ variables which are homogeneous of degree $k$. For fixed $k$ and $n \rightarrow \infty$, the lower and upper bounds differ in an essential way. The lower bound is $O\left(n^{k}\right)$ whereas the upper bound is $O\left(n^{2 k}\right)$. Integrating polynomials of degree $2 k$ with dimension $O\left(n^{2 k}\right)$, one might expect the upper bound to be tight; looking at Dvoretzky's theorem, just putting $\varepsilon=0$, one might suspect the lower bound $O\left(n^{k}\right)=O\left(n^{p / 2}\right)$ to be tight. The right order is unknown so far, except for $k=2$. The lower bound is an equality for tight designs; they have been characterized by Bannai [B] and Hogger $[\mathrm{H}]$ :

Proposition 3. Let $n, k \in \mathbb{N}$ and assume that the lower bound in (2.2) is an equality. Then the nodes $\left(x_{s}\right)_{s=1}^{N} \subseteq S^{n-1}$ and the measure $\left(\mu_{s}\right)_{s=1}^{N}$ of any design with (2.1) satisfy $\mu_{s}=1 / N$ and for any $1 \leq s \neq t \leq N$ the value $\left|\left\langle x_{s}, x_{t}\right\rangle\right|$ is a zero of a certain explicit polynomial of degree $k$ (in terms of Jacobi polynomials even/odd if $k$ is even/odd). For $k=2,\left|\left\langle x_{s}, x_{t}\right\rangle\right|=1 / \sqrt{k+2}(\mathbb{R})$ or $=1 / \sqrt{k+1}(\mathbb{C})$ for $s \neq t$ and $N=n(n+1) / 2(\mathbb{R})$ or $n^{2}(\mathbb{C})$. This is equivalent to the existence of the maximal possible number of "equiangular lines" $\mathbb{K} x_{s}$ in $\mathbb{K}^{n}$.

Designs attaining the lower bound are called tight. They do not exist for $k>5$, but there are a few cases if $k \leq 5([\mathrm{~B}],[\mathrm{H}])$ : they exist for 


$$
\begin{array}{ll}
\mathbb{K}=\mathbb{R}: \quad & k=2, n=2,3,7,23 \text { when } N=3,6,28,276 \text { (equiangular lines) } \\
& k=3, n=8,23 \text { when } n=120,2300 \\
& k=5, n=24 \text { when } N=98280 \text { (points in the Leech lattice) } \\
& n=2, \quad N=k+1 \quad\left(n \text {-the root of unity in } \mathbb{C} \simeq \mathbb{R}^{2}\right. \text { ) } \\
\mathbb{K}=\mathbb{C}: \quad & k=2, n=2,3,8 \text { when } N=4,9,64 \\
& k=3, n=4,6 \text { when } N=40,126 \\
& n=2, \quad k=2,3,5 \text { when } N=4,6,12 .
\end{array}
$$

In these cases, $\ell_{2}^{n} \rightarrow \ell_{2 k}^{N}$ imbeds isometrically and $N$ is the smallest dimension for this to hold. The examples following Proposition 1 were of this form.

To find a sequence of almost tight 4-designs in $\mathbb{K}^{n}$ with (2.1) we use sets of equiangular vectors in $\mathbb{C}^{n}$ which attain almost the maximal possible number $n^{2}$ of such vectors. We use the number-theoretic $B_{2}$-sequences employed by Rudin in his constructions of $\Lambda(4)$-sets.

Proposition $4([\mathrm{~K}])$. Let $n=p^{m}+1$, p prime. Then there exists an isometric imbedding $\ell_{2}^{n} \hookrightarrow \ell_{4}^{n^{2}+1}$ over $\mathbb{C}$.

Proof. Let $N=n^{2}-n+1$. There exist integers $0 \leq d_{1}<\cdots d_{n}<N$ such that all numbers from 1 to $N-1=n(n-1)$ occur exactly once as residue $\bmod N$ of a difference $d_{j}-d_{k}(j \neq k)$, of which there are $N-1=n(n-1)$, cf [HR]. Define

$$
x_{s}:=\frac{1}{\sqrt{n}}\left(\exp \left(\frac{2 \pi i}{N} d_{j} s\right)\right)_{j=1}^{n} \in S^{n-1}(\mathbb{C}) \subseteq \mathbb{C}^{n} ; \quad s=1, \ldots, N .
$$

These vectors are equiangular with $\left|\left\langle x_{s}, x_{t}\right\rangle\right|=\sqrt{n-1} / n$ for all $1 \leq s \neq t \leq N$ as seen by calculating $\left|\left\langle x_{s}, x_{t}\right\rangle\right|^{2}$ by using that $\sum_{j \neq k} \exp \left(\frac{2 \pi i}{N}\left(d_{j}-d_{k}\right) \Theta\right)=-1$ for all $1 \leq \Theta \leq N-1$. These vectors do not quite suffice to have equality in (2.1) with $\mu_{s}=1 / N$. However, adding the $n$ unit vectors $e_{j}=(0 \ldots 0,1, \ldots 0), j=1, \ldots, n$; i.e. letting $x_{N+j}:=e_{j}$, we have $n^{2}+1$ vectors which satisfy (2.1) if one puts in addition $\mu_{s}=\frac{n}{n+1} \frac{1}{N}$ for $1 \leq s \leq N$ and $\mu_{N+j}=\frac{n}{n+1} \frac{1}{n^{2}}$ for $1 \leq j \leq n$. There $c_{n, 2}=2 /[n(n+1)]$ for $\mathbb{K}=\mathbb{C}$. Hence $\ell_{2}^{n} \hookrightarrow \ell_{4}^{n^{2}+1}$ over $\mathbb{C}$.

These complex cubature formulas give rise to real cubature formulas of degree 4 in $m=2 n$ real variables with $M=3\left(n^{2}+1\right)=\frac{3}{4}\left(m^{2}+4\right)$ nodes as follows from the chain of imbeddings

$$
\ell_{2}^{m}(\mathbb{R})=\ell_{2}^{n}(\mathbb{C}) \hookrightarrow \ell_{4}^{n^{2}+1}(\mathbb{C})=\ell_{4}^{n^{2}+1}\left(\ell_{2}^{2}(\mathbb{R})\right) \hookrightarrow \ell_{4}^{n^{2}+1}\left(\ell_{4}^{3}(\mathbb{R})\right)=\ell_{4}^{3\left(n^{2}+1\right)}(\mathbb{R}) .
$$

Also these have only $O\left(\mathrm{~m}^{2}\right)$ nodes and thus asymptotically are of the lower asymptotic order in Proposition 2 as $m \rightarrow \infty$.

Similar constructions for $B_{k}$-sequences for $k \geq 3$ do not seem to work; one would have to add more vectors than just the unit vectors $e_{j}$. For $k=3$ one can add $O\left(n^{2}\right)$ vectors but to satisfy (2.1) some of the weights $\mu_{s}$ would have to be negative which is not allowed since $\mu$ would not define a (positive) probability measure. It is an important open problem whether the lower bound in Proposition 3 gives the right asymptotic order as $n \rightarrow \infty$ also if $k \geq 3$ is fixed. A positive answer would mean that one could put $\varepsilon=0$ in Dvoretzky's theorem for $\ell_{p}^{N}$ if $0=2 k$. 
3. Projection constants. Spherical designs and equiangular lines also show up in estimating projection constants and in the construction of spaces with maximal projection constants. Any separable, in particular any finite-dimensional Banach space $X$ may be imbedded into $\ell_{\infty}$. Take any fixed linear isometric imbedding $X \subseteq \ell_{\infty}$ and define the projection constant of $X$ by

$$
\lambda(X):=\inf \left\{\|P\| \mid P^{2}=P: \ell_{\infty} \rightarrow X \subseteq \ell_{\infty} \text { is a linear projection onto } X\right\} .
$$

This value is independent of the particular imbedding and $\ell_{\infty}$ is the "worst" superspace: if $\operatorname{dim} X<\infty$ and $X$ is isometrically imbedded into some Banach space $Y$, there is a projection $Q^{2}=Q: Y \rightarrow X \subseteq Y$ onto $X$ with $\|Q\| \leq \lambda(X)$. For $n$-dimensional Hilbert spaces, $\lambda\left(\ell_{2}^{n}\right) \sim \sqrt{\frac{2}{\pi}} \sqrt{n}(\mathbb{K}=\mathbb{R})$ and $\sim \frac{\sqrt{\pi}}{2} \sqrt{n}(\mathbb{K}=\mathbb{C})$. Kadec and Snobar [KS] showed that $\lambda(X) \leq \sqrt{n}$ holds for any $n$-dimensional space $X$. This estimate was improved in [KT1] where the following dual characterization of extremal projection constants was proved; the supremum on the right side is attained in some cases by spherical designs:

Proposition 5 ([KT1]). Let $n \in \mathbb{N}, \mathbb{K} \in\{\mathbb{R}, \mathbb{C}\}$. Then

$$
\begin{array}{r}
\sup \left\{\lambda\left(X_{n}\right) \mid \operatorname{dim} X_{n} \leq n\right\}=n \sup \left\{\sum_{s, t=1}^{N} \mu_{s} \mu_{t}\left|\left\langle x_{s}, x_{t}\right\rangle\right| \mid N \in \mathbb{N} \cup\{\infty\}, \mu_{t}>0, \sum_{t=1}^{N} \mu_{t}=1\right. \\
\left.\operatorname{Id}_{n}=n \sum_{t=1}^{N} \mu_{t} x_{t} \otimes x_{t}\right\}
\end{array}
$$

Both suprema are in fact attained as maxima.

The proof uses the trace-dual characterization of projection constants in terms of 1-summing norms; $\mu=\left(\mu_{t}\right)$ is the corresponding Pietsch measure. Since

$$
n \sum_{s, t=1}^{N} \mu_{s} \mu_{t}\left|\left\langle x_{s}, x_{t}\right\rangle\right| \leq n\left(\sum_{s, t=1}^{N} \mu_{s} \mu_{t}\left|\left\langle x_{s}, x_{t}\right\rangle\right|^{2}\right)^{1 / 2}=\sqrt{n}
$$

under the constraints on $\left(x_{t}, \mu_{t}\right)$, the Kadec-Snobar result is an immediate consequence of (3.1). An improvement can be derived by estimating $|u| \leq a+b u^{2}-c u^{4}$ for appropriate $a, b, c>0$ with $u=\left\langle x_{s}, x_{t}\right\rangle$ and by using the Sidelnikov inequality mentioned in the proof of Proposition 1. This way one proves

TheOREM $6([\mathrm{KT} 1])$. Let $n=\mathbb{N}$. Then for any $n$-dimensional Banach space $X_{n}$

$$
\lambda\left(X_{n}\right) \leq G(n):=\left\{\begin{array}{ll}
(2+(n-1) \sqrt{n+2}) /(n+1), & \mathbb{K}=\mathbb{R} \\
(1+(n-1) \sqrt{n+1}) / n, & \mathbb{K}=\mathbb{C}
\end{array}\right\}<\sqrt{n} .
$$

There is $X_{n}, \operatorname{dim} X_{n}=n$, with $\lambda\left(X_{n}\right)=G(n)$ if and only if there exist $N(n)$ equiangular lines in $\mathbb{K}^{n}$ where

$$
N(n)=\left\{\begin{array}{cc}
n(n+1) / 2, & \mathbb{K}=\mathbb{R} \\
n^{2}, & \mathbb{K}=\mathbb{C}
\end{array}\right\}
$$

i.e. if and only if there is a tight 4-design in $S^{n-1}(\mathbb{K}) \subseteq \mathbb{K}^{n}$.

If $\left(x_{s}, \mu_{s}=\frac{1}{N(n)}\right)_{s=1}^{N(n)}$ is such a tight design, the norm of $X_{n}=\left(\mathbb{K}^{n},\|\cdot\|\right)$ with $\lambda\left(X_{n}\right)=G(n)$ may be defined as $\|x\|=\max _{1 \leq s \leq N(n)}\left|\left\langle x, x_{s}\right\rangle\right|$. Thus $X_{n} \subset \ell_{\infty}^{N(n)}$ is very 
badly complemented. Taking the $\ell_{4}$-norm, instead of the $\ell_{\infty}$-norm, however, one gets isometrically a Hilbertian subspace of $\ell_{4}^{N(n)}$ onto which in addition uniformly bounded projections exist. For $\mathbb{K}=\mathbb{R}$ and $n=2,3$ spaces with maximal projection constants derived from this are those whose unit balls are the regular hexagon and the regular dodecahedron. However, in general spaces with extremal projection constants are not unique since a corresponding imbedding into $\ell_{1}^{N}$ instead of $\ell_{\infty}^{N}$ may yield a different space with the same projection constant:

Proposition 7 ([KT2]). Let $X_{\infty} \subseteq \ell_{\infty}^{N}, \operatorname{dim} X_{\infty}=n$ be such that $\lambda\left(X_{\infty}\right)=\sup \{\lambda(Y) \mid$ $\operatorname{dim} Y=n\}$. Then there exists a probability measure $\mu=\left(\mu_{s}\right)_{s=1}^{N}$ on $\{1, \ldots, N\}$ such that when the same space $X_{\infty}$ is considered as a subspace of $\ell_{1}^{N}(\mu)$, then written as $X_{1} \subseteq \ell_{1}^{N}(\mu)$, one has $\lambda\left(X_{\infty}\right)=\lambda\left(X_{1}\right)$.

For $\mathbb{K}=\mathbb{R}$ and $n=2$ one gets the hexagonal space in both cases. In general, however, the spaces $X_{\infty}$ and $X_{1}$ are very different. For $\mathbb{K}=\mathbb{R}$ and $n=3, x_{s}=$ diagonals of the icosahedron, $X_{\infty}$ is the space with dodecahedron unit ball and $X_{1}$ the space whose unit ball is the dodecahedron intersected with the $\phi$-multiple of the face-centrally inscribed icosahedron, $\phi=(1+\sqrt{5}) / 2$. The second unit ball has as its faces 12 pentagons-as the dodecahedron-plus 20 triangles, cf. [KT2].

Taking the almost maximal number of equiangular vectors $x_{s}$ given by $(2.3)$ in $\mathbb{C}^{n}$ where $n=p^{m}+1$ and $s=1, \ldots, N:=n^{2}-n+1$, we may define a space $X_{\infty}=\left(\mathbb{C}^{n},\|\cdot\|\right) \subseteq$ $\ell_{\infty}^{N}$ by letting

$$
\|x\|:=\sup _{1 \leq s \leq N}\left|\left\langle x, x_{s}\right\rangle\right|
$$

In this case, one can check that $X_{\infty}$ has almost extremal projection constant. More precisely, cf. [KT3],

$$
\lambda\left(X_{\infty}\right) \geq G(n)-1 / n^{3 / 2} \geq \sqrt{n}-1 / \sqrt{n} .
$$

In particular, $\lim _{n \rightarrow \infty} \lambda\left(X_{\infty}\right) / \sqrt{n}=1$ when $n$ is a sequence of integers of the form $n=p^{m}+1$ tending to infinity. The spaces $X_{\infty}$ also have extremal distance to $\ell_{2}^{n}$ and extremal unconditional basis constant $u b c$, i.e.

$$
d\left(X_{\infty}, \ell_{2}^{n}\right)=\sqrt{n}, \quad u b c\left(X_{\infty}\right)=\sqrt{n} .
$$

As subspaces of $\ell_{p}^{N}$, denoted $X_{p}$ they have uniformly bounded Banach-Mazur distance to $\ell_{2}^{n}$ provided that $1 \leq p \leq 4$; onto these spaces, as subspaces of $\ell_{p}^{N}$, there are also uniformly bounded projections. For these facts see [KT2], [KT3].

Further beautiful applications of spherical designs to the Borsuk and Knaster conjectures are given in papers of Hinrichs and Richter [HR1], [HR2].

\section{References}

[B] E. Bannai, On extremal sets in the sphere and other metric spaces, in: London Math. Soc. Lecture Notes Ser. 131, Cambridge Univ. Press, 1986, 13-38.

[D] A. Dvoretzky, Some results on convex bodies and Banach spaces, in: Proc. Symp. on Linear Spaces, Jerusalem, 1961, 123-160. 
[DGS] P. Delsarte, J. M. Goethals and J. J. Seidel, Spherical codes and designs, Geometriae Dedicata 6 (1977), 363-388.

[DJP] F. Delbaen, H. Jarchow and A. Pełczyński, Subspaces of $L_{p}$ isometric to subspaces of $\ell_{p}$, Positivity 2 (1998), 339-367.

[FLM] T. Figiel, J. Lindenstrauss and V. D. Milman, The dimensions of almost spherical sections of convex bodies, Acta Math. 139 (1977), 53-94.

[G] E. D. Gluskin, The diameter of the Minkowski compactum is roughly equal to n, Funct. Anal. Appl. 15 (1981), 72-73.

[H] S. G. Hoggar, t-designs in projective spaces, European J. Combin. 3 (1982), 233-254.

[HR] H. Halberstam and K. Roth, Sequences, Springer, 1982.

[HR1] A. Hinrichs and Ch. Richter, New sets with large Borsuk numbers, preprint, Jena, 2002.

[HR2] A. Hinrichs and Ch. Richter, New counterexamples to Knaster's conjecture, preprint, Jena, 2003.

[KS] I. M. Kadec and M. G. Snobar, Certain functionals on the Minkowski compactum, Math. Notes 10 (1971), 694-696 [English transl.].

[Ka] B. S. Kashin, Sections of some finite-dimensional sets and classes of smooth functions, Izv. Akad. Nauk SSSR Ser. Mat. 41 (1977), 334-351.

[K] H. König, Isometric imbeddings of Euclidean spaces onto finite-dimensional $\ell_{p}$-spaces, in: Banach Center Publ. 34, Inst. Math., Polish Acad. Sci., 1995, 79-87.

[KT1] H. König and N. Tomczak-Jaegermann, Norms of minimal projections, J. Funct. Anal. 119 (1994), 253-280.

[KT2] H. König and N. Tomczak-Jaegermann, Spaces with maximal projection constants, Studia Math. 159 (2003), 357-372.

[KT3] H. König and N. Tomczak-Jaegermann, Bounds for projection constants and 1-summing norms, Trans. AMS 320 (1990), 799-823.

[L] Yu. Lyubich, On the boundary spectrum of a contraction in Minkowski spaces, Siberian Math. J. 11 (1970), 271-279 [English transl.].

[LV] Yu. Lyubich and L. Vaserstein, Isometric imbeddings between classical Banach spaces, cubature formulas and spherical designs, Geometriae Dedicata 47 (1993), 327-362.

[M] V. D. Milman, A few observations on the connections between local theory and some other fields, in: Geometric Aspects of Functional Analysis, Lect. Notes in Math. 1317, Springer, 1988, 283-289.

[MS] V. D. Milman and G. Schechtman, Asymptotic Theory of Finite Dimensional Normal Spaces, Lecture Notes in Math. 1200, Springer, 1986.

[R] B. Reznick, Sums of even powers of real linear forms, Mem. AMS 96 (1992), no. 463. 